\title{
Symmetric diophantine systems revisited
}

by

\section{Ajai Choudhry (New Delhi)}

1. Introduction. In this paper we consider symmetric diophantine systems consisting of two or three simultaneous symmetric equations of the type

$$
f\left(x_{1}, x_{2}, x_{3}\right)=f\left(y_{1}, y_{2}, y_{3}\right),
$$

where $f\left(x_{1}, x_{2}, x_{3}\right)$ is a linear, quadratic or cubic form in the three variables $x_{1}, x_{2}$, and $x_{3}$. The author had given solutions of several such diophantine systems in an earlier paper [1]. The present paper therefore focuses on those systems that cannot be solved by the methods given earlier. Throughout the paper we shall use $L$ 's, $Q$ 's and $C$ 's to denote linear, quadratic and cubic forms in the variables indicated. Further, all the forms occurring in the paper will be assumed to be defined over the field $\mathbb{Q}$ of rational numbers.

When the given diophantine system consists of a pair of simultaneous equations of type (1.1) of degrees not exceeding 3, except for the case when both equations are cubic, any other such diophantine system can be solved by the methods described in [1]. In this paper we consider the diophantine system consisting of two simultaneous symmetric equations, that is, the system

$$
\left\{\begin{array}{l}
C_{1}\left(x_{1}, x_{2}, x_{3}\right)=C_{1}\left(y_{1}, y_{2}, y_{3}\right), \\
C_{2}\left(x_{1}, x_{2}, x_{3}\right)=C_{2}\left(y_{1}, y_{2}, y_{3}\right),
\end{array}\right.
$$

and we obtain a necessary and sufficient condition for the solvability of this system.

When the given diophantine system consists of three simultaneous equations of type (1.1) of degrees $\leq 2$, the methods described in [1] yield the complete solution if the system consists of two linear and one quadratic equation, or one linear and two quadratic equations. Further, the complete solution of a system of three linear equations of type (1.1) is readily obtained.

2000 Mathematics Subject Classification: Primary 11D25. 
In this paper we therefore consider the following diophantine systems:

(VIII)

$$
\begin{aligned}
& \left\{\begin{array}{c}
L_{1}\left(x_{1}, x_{2}, x_{3}\right)=L_{1}\left(y_{1}, y_{2}, y_{3}\right), \\
L_{2}\left(x_{1}, x_{2}, x_{3}\right)=L_{2}\left(y_{1}, y_{2}, y_{3}\right), \\
C\left(x_{1}, x_{2}, x_{3}\right)=C\left(y_{1}, y_{2}, y_{3}\right),
\end{array}\right. \\
& \left\{\begin{array}{l}
L\left(x_{1}, x_{2}, x_{3}\right)=L\left(y_{1}, y_{2}, y_{3}\right), \\
Q\left(x_{1}, x_{2}, x_{3}\right)=Q\left(y_{1}, y_{2}, y_{3}\right), \\
C\left(x_{1}, x_{2}, x_{3}\right)=C\left(y_{1}, y_{2}, y_{3}\right),
\end{array}\right. \\
& \left\{\begin{array}{c}
L\left(x_{1}, x_{2}, x_{3}\right)=L\left(y_{1}, y_{2}, y_{3}\right), \\
C_{1}\left(x_{1}, x_{2}, x_{3}\right)=C_{1}\left(y_{1}, y_{2}, y_{3}\right), \\
C_{2}\left(x_{1}, x_{2}, x_{3}\right)=C_{2}\left(y_{1}, y_{2}, y_{3}\right),
\end{array}\right. \\
& \left\{\begin{array}{l}
Q_{1}\left(x_{1}, x_{2}, x_{3}\right)=Q_{1}\left(y_{1}, y_{2}, y_{3}\right), \\
Q_{2}\left(x_{1}, x_{2}, x_{3}\right)=Q_{2}\left(y_{1}, y_{2}, y_{3}\right), \\
Q_{3}\left(x_{1}, x_{2}, x_{3}\right)=Q_{3}\left(y_{1}, y_{2}, y_{3}\right),
\end{array}\right. \\
& \left\{\begin{array}{c}
Q_{1}\left(x_{1}, x_{2}, x_{3}\right)=Q_{1}\left(y_{1}, y_{2}, y_{3}\right), \\
Q_{2}\left(x_{1}, x_{2}, x_{3}\right)=Q_{2}\left(y_{1}, y_{2}, y_{3}\right), \\
C\left(x_{1}, x_{2}, x_{3}\right)=C\left(y_{1}, y_{2}, y_{3}\right),
\end{array}\right. \\
& \left\{\begin{aligned}
Q\left(x_{1}, x_{2}, x_{3}\right) & =Q\left(y_{1}, y_{2}, y_{3}\right), \\
C_{1}\left(x_{1}, x_{2}, x_{3}\right) & =C_{1}\left(y_{1}, y_{2}, y_{3}\right), \\
C_{2}\left(x_{1}, x_{2}, x_{3}\right) & =C_{2}\left(y_{1}, y_{2}, y_{3}\right),
\end{aligned}\right. \\
& \left\{\begin{array}{l}
C_{1}\left(x_{1}, x_{2}, x_{3}\right)=C_{1}\left(y_{1}, y_{2}, y_{3}\right), \\
C_{2}\left(x_{1}, x_{2}, x_{3}\right)=C_{2}\left(y_{1}, y_{2}, y_{3}\right), \\
C_{3}\left(x_{1}, x_{2}, x_{3}\right)=C_{3}\left(y_{1}, y_{2}, y_{3}\right) .
\end{array}\right.
\end{aligned}
$$

As all the diophantine systems considered in this paper consist of homogeneous equations only, any rational solution can be multiplied throughout by a suitable constant to obtain a solution in integers. It therefore suffices if we consider the solvability of these systems in rational numbers. Further, for all of these diophantine systems, we will generally consider a solution to be non-trivial if $x_{j} \neq y_{j}$ for some $j \in\{1,2,3\}$. We now consider, one by one, each of the diophantine systems mentioned above.

2. The diophantine system (I). We first consider the system (I), that is,

$$
\begin{aligned}
& C_{1}\left(x_{1}, x_{2}, x_{3}\right)=C_{1}\left(y_{1}, y_{2}, y_{3}\right), \\
& C_{2}\left(x_{1}, x_{2}, x_{3}\right)=C_{2}\left(y_{1}, y_{2}, y_{3}\right) .
\end{aligned}
$$


A particular case, arising from an unsolved problem mentioned by Guy [4, p. 142], is the system

$$
\begin{aligned}
x_{1}^{3}+x_{2}^{3}+x_{3}^{3} & =y_{1}^{3}+y_{2}^{3}+y_{3}^{3}, \\
x_{1} x_{2} x_{3} & =y_{1} y_{2} y_{3},
\end{aligned}
$$

for which several parametric solutions have already been obtained ([2], $[3$, p. 101]).

We will first show that if one non-trivial solution of (I) is known, it can, in general, be used to derive another non-trivial solution. Next we will obtain a necessary and sufficient condition for the solvability of this system. A specific example, together with an additional symmetric linear equation, illustrating the application of the solvability condition for this system, will be given in Section 5 .

Let $x_{j}=\xi_{j}, y_{j}=\eta_{j}, j=1,2,3$, be a known non-trivial solution of the system (I) so that $C_{i}\left(\xi_{1}, \xi_{2}, \xi_{3}\right)=C_{i}\left(\eta_{1}, \eta_{2}, \eta_{3}\right), i=1,2$. To obtain another non-trivial solution, we write

$$
x_{j}=\xi_{j} \theta+X_{j}, \quad y_{j}=\eta_{j} \theta+X_{j}, \quad j=1,2,3,
$$

where $X_{1}, X_{2}, X_{3}$ and $\theta$ are arbitrary parameters with $\theta \neq 0$. Substituting these values of $x_{j}, y_{j}, j=1,2,3$, in (2.1) and (2.2), transposing all the terms to one side, and removing the factor $\theta$ from both equations, we get the equations

$$
\begin{aligned}
\frac{\theta}{2}\left\{\left(\sum_{j=1}^{3} \xi_{j}\right.\right. & \left.\left.\frac{\partial}{\partial X_{j}}\right)^{2}-\left(\sum_{j=1}^{3} \eta_{j} \frac{\partial}{\partial X_{j}}\right)^{2}\right\} C_{i}\left(X_{1}, X_{2}, X_{3}\right) \\
+ & \left\{\sum_{j=1}^{3}\left(\xi_{j}-\eta_{j}\right) \frac{\partial}{\partial X_{j}}\right\} C_{i}\left(X_{1}, X_{2}, X_{3}\right)=0, \quad i=1,2 .
\end{aligned}
$$

We can find a new non-trivial solution of (I) if the two values of $\theta$ obtained from the two equations (2.5) are equal. These two values may be written as $Q_{i}\left(X_{1}, X_{2}, X_{3}\right) / L_{i}\left(X_{1}, X_{2}, X_{3}\right), i=1,2$, where the forms $L_{i}\left(X_{1}, X_{2}, X_{3}\right)$, $Q_{i}\left(X_{1}, X_{2}, X_{3}\right), i=1,2$, are determined by the two equations (2.5), and for the two values to be equal, we must choose $X_{1}, X_{2}, X_{3}$ such that

$$
L_{1}\left(X_{1}, X_{2}, X_{3}\right) Q_{2}\left(X_{1}, X_{2}, X_{3}\right)=L_{2}\left(X_{1}, X_{2}, X_{3}\right) Q_{1}\left(X_{1}, X_{2}, X_{3}\right) .
$$

Equation (2.6) represents a cubic curve in the projective plane, and a rational point on it can be determined by solving the two linear equations $L_{i}\left(X_{1}, X_{2}, X_{3}\right)=0, i=1,2$. This rational point can, in general, be used to obtain another rational point on the curve (2.6), and hence we can derive another non-trivial solution of the system (I).

We will now obtain a necessary and sufficient condition for the solvability of (I). We note that if the simultaneous equations $C_{i}\left(x_{1}, x_{2}, x_{3}\right)=0, i=1,2$, 
have an integer solution, say, $x_{j}=\xi_{j}, j=1,2,3$, then a solution of (I) is given by $x_{j}=\xi_{j}, y_{j}=k \xi_{j}, j=1,2,3$, where $k$ is an arbitrary rational number. All such solutions of (I) are readily determined. For all non-trivial solutions, we must have $x_{j} \neq y_{j}$ for some $j \in\{1,2,3\}$, and we assume at present that $x_{1} \neq y_{1}$. Further, the relations $x_{1} / y_{1}=x_{2} / y_{2}=x_{3} / y_{3}$ imply that $y_{j}=k x_{j}, j=1,2,3$, and all such solutions have already been determined. Thus for other non-trivial solutions, we cannot have both $x_{1} y_{2}-$ $x_{2} y_{1}=0$ and $x_{1} y_{3}-x_{3} y_{1}=0$; we therefore assume that $x_{1} y_{2}-x_{2} y_{1} \neq 0$. To obtain solutions of (I) such that $x_{1} \neq y_{1}$ and $x_{1} y_{2}-x_{2} y_{1} \neq 0$, we write

$$
\begin{array}{ll}
x_{1}=a_{1} X_{1}+a_{1} m X_{2}, & y_{1}=-a_{1} X_{1}+a_{1} m X_{2}, \\
x_{2}=a_{2} X_{1}+\left(a_{2} m+1\right) X_{2}, & y_{2}=-a_{2} X_{1}+\left(a_{2} m+1\right) X_{2}, \\
x_{3}=a_{3} X_{1}+a_{3} m X_{2}+X_{3}, & y_{3}=-a_{3} X_{1}+a_{3} m X_{2}+X_{3},
\end{array}
$$

where $a_{1}, a_{2}, a_{3}, X_{1}, X_{2}, X_{3}$ and $m$ are arbitrary parameters with $X_{1} \neq 0$, $X_{2} \neq 0$. With these values of $x_{j}, y_{j}$, equations (2.1) and (2.2) reduce to

$$
\begin{aligned}
2 C_{i}\left(a_{1}, a_{2}, a_{3}\right) & X_{1}^{2}+6 C_{i}\left(a_{1}, a_{2}, a_{3}\right) m^{2} X_{2}^{2} \\
& +2 m L_{i}\left(X_{2}, X_{3}\right) X_{2}+Q_{i}\left(X_{2}, X_{3}\right)=0, \quad i=1,2,
\end{aligned}
$$

where the forms $L_{i}\left(X_{2}, X_{3}\right), Q_{i}\left(X_{2}, X_{3}\right), i=1,2$, are given by

$$
\begin{aligned}
L_{i}\left(X_{2}, X_{3}\right) & =\sum_{r=2}^{3} X_{r} \frac{\partial}{\partial x_{r}}\left(\sum_{j=1}^{3} a_{j} \frac{\partial}{\partial x_{j}}\right)^{2} C_{i}, \\
Q_{i}\left(X_{2}, X_{3}\right) & =\left(X_{2} \frac{\partial}{\partial x_{2}}+X_{3} \frac{\partial}{\partial x_{3}}\right)^{2}\left(\sum_{j=1}^{3} a_{j} \frac{\partial}{\partial x_{j}}\right) C_{i} .
\end{aligned}
$$

A rational solution of the simultaneous equations (2.8) will exist if the following condition, obtained by eliminating $m$ from the two equations (2.8), is satisfied:

$$
\begin{aligned}
4\left\{C_{2}\left(a_{1}, a_{2}, a_{3}\right) L_{1}\left(X_{2}, X_{3}\right)-C_{1}\left(a_{1}, a_{2}, a_{3}\right) L_{2}\left(X_{2}, X_{3}\right)\right\}^{2} X_{1}^{2} \\
=-3\left\{C_{1}\left(a_{1}, a_{2}, a_{3}\right) Q_{2}\left(X_{2}, X_{3}\right)-C_{2}\left(a_{1}, a_{2}, a_{3}\right) Q_{1}\left(X_{2}, X_{3}\right)\right\}^{2} \\
\quad-2\left\{L_{1}\left(X_{2}, X_{3}\right) Q_{2}\left(X_{2}, X_{3}\right)-L_{2}\left(X_{2}, X_{3}\right) Q_{1}\left(X_{2}, X_{3}\right)\right\} \\
\quad \times\left\{C_{2}\left(a_{1}, a_{2}, a_{3}\right) L_{1}\left(X_{2}, X_{3}\right)-C_{1}\left(a_{1}, a_{2}, a_{3}\right) L_{2}\left(X_{2}, X_{3}\right)\right\} .
\end{aligned}
$$

When rational values of $a_{1}, a_{2}, a_{3}, X_{1}, X_{2}$, and $X_{3}$ are chosen such that (2.10) is satisfied, the two equations (2.8) are simultaneously satisfied by the same rational value of $m$, and substituting these values of $a_{1}, a_{2}, a_{3}, X_{1}, X_{2}, X_{3}$ and $m$ in (2.7), we get a rational solution of the diophantine system (I).

We have thus shown that a sufficient condition for the solvability of (I) with $x_{1} \neq y_{1}$ and $x_{1} y_{2}-x_{2} y_{1} \neq 0$ is that there exist rational values of $a_{1}, a_{2}, a_{3}, X_{1}, X_{2}$, and $X_{3}$ such that (2.10) is satisfied. We will now show 
that this condition is also necessary. Let $x_{j}=\xi_{j}, y_{j}=\eta_{j}, j=1,2,3$, be any arbitrary solution of (I) with $x_{1} \neq y_{1}$ and $x_{1} y_{2}-x_{2} y_{1} \neq 0$ so that $C_{i}\left(\xi_{1}, \xi_{2}, \xi_{3}\right)=C_{i}\left(\eta_{1}, \eta_{2}, \eta_{3}\right), i=1,2$, and also $\xi_{1} \neq \eta_{1}, \xi_{1} \eta_{2}-\xi_{2} \eta_{1} \neq 0$. For each $j=1,2,3$, we replace $x_{j}$ by $\xi_{j}$ and $y_{j}$ by $\eta_{j}$ in (2.7), and solve the resulting equations for $a_{1}, a_{2}, a_{3}, X_{1}, X_{2}, X_{3}$ and $m$ to get

$$
\begin{gathered}
a_{1}=\xi_{1}-\eta_{1}, \quad a_{2}=\xi_{2}-\eta_{2}, \quad a_{3}=\xi_{3}-\eta_{3}, \quad m=\frac{\xi_{1}+\eta_{1}}{2\left(\xi_{1} \eta_{2}-\xi_{2} \eta_{1}\right)}, \\
X_{1}=\frac{1}{2}, \quad X_{2}=\frac{\xi_{1} \eta_{2}-\xi_{2} \eta_{1}}{\xi_{1}-\eta_{1}}, \quad X_{3}=\frac{\xi_{1} \eta_{3}-\xi_{3} \eta_{1}}{\xi_{1}-\eta_{1}},
\end{gathered}
$$

where we note that neither $X_{1}$ nor $X_{2}$ is 0 . Since $C_{i}\left(\xi_{1}, \xi_{2}, \xi_{3}\right)=C_{i}\left(\eta_{1}, \eta_{2}, \eta_{3}\right)$, $i=1,2$, it follows that the values given by (2.11) satisfy (2.8) and hence also (2.10). This shows that (2.10) is a necessary and sufficient condition for the existence of non-trivial solutions of (I) with $x_{1} \neq y_{1}$ and $x_{1} y_{2}-x_{2} y_{1} \neq 0$.

By substituting

$$
X_{1}=\sigma X_{3} /\left[2\left\{C_{2}\left(a_{1}, a_{2}, a_{3}\right) L_{1}(\varrho, 1)-C_{1}\left(a_{1}, a_{2}, a_{3}\right) L_{2}(\varrho, 1)\right\}\right], \quad X_{2}=\varrho X_{3},
$$

condition (2.10) may be written as follows:

$$
\sigma^{2}=\sum_{r=0}^{4} \phi_{r}\left(a_{1}, a_{2}, a_{3}\right) \varrho^{r}
$$

where $\phi_{r}\left(a_{1}, a_{2}, a_{3}\right), r=0, \ldots, 4$, are forms of degree 8 in $a_{1}, a_{2}$ and $a_{3}$. We note that in view of our assumptions $x_{1} \neq y_{1}$ and $x_{1} y_{2}-x_{2} y_{1} \neq 0$, for any given diophantine system, we will actually have six conditions of the type (2.12), and the solution of any one of these equations will lead to a solution of the given system. To solve an equation of type (2.12), we assign numerical trial values to the parameters $a_{1}, a_{2}, a_{3}$ when the quartic equation (2.12) represents an elliptic curve, and so we can efficiently perform a search for rational points on this curve using a computer program such as APECS, a package written in Maple specifically for working with elliptic curves. If for some numerical values of $a_{1}, a_{2}, a_{3}$, we can find a rational point on the elliptic curve (2.12), and this curve is of positive rank, we can find infinitely many rational points on this curve, and hence obtain infinitely many solutions of the system (I).

An alternative necessary and sufficient condition for the solvability of (I) with $x_{1} \neq y_{1}$ and $x_{1} y_{2}-x_{2} y_{1} \neq 0$ may be obtained by writing

$$
x_{3}=p x_{1}+q x_{2}, \quad y_{3}=p y_{1}+q y_{2},
$$

so that the system (I) reduces to

$$
\left\{\begin{array}{l}
C_{1}^{\prime}\left(x_{1}, x_{2}\right)=C_{1}^{\prime}\left(y_{1}, y_{2}\right), \\
C_{2}^{\prime}\left(x_{1}, x_{2}\right)=C_{2}^{\prime}\left(y_{1}, y_{2}\right),
\end{array}\right.
$$


where the coefficients of the binary cubic forms $C_{i}^{\prime}\left(x_{1}, x_{2}\right), i=1,2$, are polynomials in $p$ and $q$. To solve (I), it is clearly sufficient to solve $\left(\mathrm{I}^{\prime}\right)$ for some rational values of $p$ and $q$. Conversely, if (I) has a solution with $x_{1} y_{2}-x_{2} y_{1} \neq 0$, there exist rational numbers $p$ and $q$ such that the relations (2.13) are satisfied, and hence there exists a solution of $\left(\mathrm{I}^{\prime}\right)$. A necessary and sufficient condition for the solvability of $\left(\mathrm{I}^{\prime}\right)$, and hence of (I), obtained by applying a theorem given by Choudhry [3, pp. 94-95], may be written as

$$
\sigma^{2}=\sum_{r=0}^{4} \psi_{r}(p, q) \varrho^{r}
$$

where $\psi_{r}(p, q), r=0, \ldots, 4$, are polynomials of degree 8 in $p, q$ determined by the aforesaid theorem, and there must exist rational numbers $p, q, \varrho$ and $\sigma$ satisfying (2.14). While this condition is similar to the first condition (2.12) obtained above, we prefer the condition (2.12) as it can be applied, with minor changes, to other diophantine systems such as (IV).

Finally, we note that if $C_{2}\left(x_{1}, x_{2}, x_{3}\right)=x_{1} x_{2} x_{3}$, parametric solutions of (I) can easily be obtained by writing $x_{1}=p X_{1}, x_{2}=q X_{2}, x_{3}=X_{1}+X_{2}$, $y_{1}=p Y_{1}, y_{2}=q Y_{2}, y_{3}=Y_{1}+Y_{2}$, when the system reduces to

$$
\begin{aligned}
C\left(X_{1}, X_{2}\right) & =C\left(Y_{1}, Y_{2}\right), \\
X_{1} X_{2}\left(X_{1}+X_{2}\right) & =Y_{1} Y_{2}\left(Y_{1}+Y_{2}\right),
\end{aligned}
$$

and a solution of this system for arbitrary $p$ and $q$ is obtained by applying Lemma 1 given in [3, p. 93].

3. The diophantine system (II). We now consider the system (II), that is,

$$
\begin{aligned}
L_{1}\left(x_{1}, x_{2}, x_{3}\right) & =L_{1}\left(y_{1}, y_{2}, y_{3}\right), \\
L_{2}\left(x_{1}, x_{2}, x_{3}\right) & =L_{2}\left(y_{1}, y_{2}, y_{3}\right), \\
C\left(x_{1}, x_{2}, x_{3}\right) & =C\left(y_{1}, y_{2}, y_{3}\right) .
\end{aligned}
$$

The two linear equations (3.1) and (3.2) may be solved to obtain

$$
y_{j}=x_{j}+\xi_{j} m, \quad j=1,2,3,
$$

where $\xi_{j}$ are integers and $m$ is an arbitrary parameter. Substituting these values of $y_{j}, j=1,2,3$, in (3.3), and removing the factor $m$, we get a homogeneous quadratic equation of the type

$$
Q\left(x_{1}, x_{2}, x_{3}, m\right)=0,
$$

in four variables $x_{1}, x_{2}, x_{3}$ and $m$. Necessary and sufficient conditions for the solvability of (3.5) are well known [5, pp. 49-51], and when these conditions are satisfied, its complete solution can be effectively determined. If (3.5) has no non-zero solutions, the system (II) has only the trivial solutions 
$x_{j}=y_{j}, j=1,2,3$, corresponding to $m=0$. If, however, (3.5) has a nonzero solution, we can find its complete solution in parametric terms, and hence we obtain the complete solution of the system (II).

We also note that when $C\left(x_{1}, x_{2}, x_{3}\right)=x_{1} x_{2} x_{3}$, equation (3.3) may be replaced by the three linear equations

$$
x_{1}=\alpha_{1} y_{1}, \quad x_{2}=\alpha_{2} y_{2}, \quad \alpha_{1} \alpha_{2} x_{3}=y_{3},
$$

where $\alpha_{1}, \alpha_{2}$ are arbitrary rational parameters, and the complete solution of the diophantine system may be found by solving the five linear equations (3.1), (3.2) and (3.6) in the six variables $x_{1}, x_{2}, x_{3}, y_{1}, y_{2}$ and $y_{3}$.

4. The diophantine system (III). We next consider the system (III), that is,

$$
\begin{aligned}
& L\left(x_{1}, x_{2}, x_{3}\right)=L\left(y_{1}, y_{2}, y_{3}\right), \\
& Q\left(x_{1}, x_{2}, x_{3}\right)=Q\left(y_{1}, y_{2}, y_{3}\right), \\
& C\left(x_{1}, x_{2}, x_{3}\right)=C\left(y_{1}, y_{2}, y_{3}\right) .
\end{aligned}
$$

A parametric solution of this system is given in [1, pp. 297-298]. This solution is, however, not complete. We now show that in cases where the cubic form $C\left(x_{1}, x_{2}, x_{3}\right)$ is a product of three linearly independent linear forms in the variables $x_{1}, x_{2}, x_{3}$, or can effectively be replaced by another cubic form that is a product of three such linear forms, it is possible to obtain the complete solution of the above diophantine system. In such cases, the system may be reduced, by a suitable invertible linear transformation, to the following system $\left(\mathrm{III}^{\prime}\right)$ :

$$
\begin{aligned}
L_{1}\left(X_{1}, X_{2}, X_{3}\right) & =L_{1}\left(Y_{1}, Y_{2}, Y_{3}\right), \\
Q_{1}\left(X_{1}, X_{2}, X_{3}\right) & =Q_{1}\left(Y_{1}, Y_{2}, Y_{3}\right), \\
X_{1} X_{2} X_{3} & =Y_{1} Y_{2} Y_{3} .
\end{aligned}
$$

All solutions of this system with $X_{1} X_{2} X_{3}=Y_{1} Y_{2} Y_{3}=0$ are readily obtained by solving equations (4.4) and (4.5) together with two linear equations $X_{u}=0, u \in\{1,2,3\}, Y_{v}=0, v \in\{1,2,3\}$. This gives us a maximum of nine two-parameter solutions which together give the complete non-trivial solution of $\left(\mathrm{III}^{\prime}\right)$ with $X_{1} X_{2} X_{3}=0$.

We will now obtain all non-trivial solutions of (III') with $X_{1} X_{2} X_{3} \neq 0$. There is no loss of generality in assuming that one of the variables, say $X_{3}$, is not identically the same as the linear form $L_{1}\left(X_{1}, X_{2}, X_{3}\right)$. The complete non-trivial solution of (III') with $X_{1} X_{2} X_{3} \neq 0$ and $X_{3}=Y_{3}$ is obtained by solving (4.4), (4.5) together with the equations $X_{1} X_{2}=Y_{1} Y_{2}, X_{3}=Y_{3}$ as described in [1, p. 293]. 
We will now obtain the complete non-trivial solution of (III') with $X_{1} X_{2} X_{3} \neq 0$ and $X_{3} \neq Y_{3}$. To obtain such solutions, we write

$$
\begin{array}{ll}
X_{1}=(\lambda \mu+1) \alpha_{1}, & Y_{1}=(\mu+1) \alpha_{1}, \\
X_{2}=\alpha_{2}, & Y_{2}=(\lambda \mu+1) \alpha_{2}, \\
X_{3}=(\mu+1) \alpha_{3}, & Y_{3}=\alpha_{3},
\end{array}
$$

where $\alpha_{1}, \alpha_{2}, \alpha_{3}$ are arbitrary non-zero integer parameters, while $\lambda$ and $\mu$ are arbitrary rational parameters with $\mu \neq 0$. With these values of $X_{j}, Y_{j}$, $j=1,2,3$, equation (4.6) is identically satisfied while equations (4.4) and (4.5) reduce, respectively, to the following equations:

$$
L_{1}\left((\lambda-1) \alpha_{1},-\lambda \alpha_{2}, \alpha_{3}\right)=0,
$$

and

$$
\begin{aligned}
\mu\left\{Q_{1}\left(\lambda \alpha_{1}, 0, \alpha_{3}\right)-\right. & \left.Q_{1}\left(\alpha_{1}, \lambda \alpha_{2}, 0\right)\right\}+\left(\frac{\partial Q_{1}}{\partial X_{1}}\right)_{X_{j}=\alpha_{j}}(\lambda-1) \alpha_{1} \\
& -\left(\frac{\partial Q_{1}}{\partial X_{2}}\right)_{X_{j}=\alpha_{j}} \lambda \alpha_{2}+\left(\frac{\partial Q_{1}}{\partial X_{3}}\right)_{X_{j}=\alpha_{j}} \alpha_{3}=0 .
\end{aligned}
$$

Equation (4.8) is a linear equation in $\lambda$ which has a unique solution $\lambda_{0}$ given by $\lambda_{0}=L_{1}\left(\alpha_{1}, 0,-\alpha_{3}\right) / L_{1}\left(\alpha_{1},-\alpha_{2}, 0\right)$. With $\lambda=\lambda_{0}$, we solve equation (4.9) for $\mu$ to get a unique solution $\mu_{0}$ which may be written as $\mu_{0}=F_{1}\left(\alpha_{1}, \alpha_{2}, \alpha_{3}\right) / F_{2}\left(\alpha_{1}, \alpha_{2}, \alpha_{3}\right)$, where $F_{i}\left(\alpha_{1}, \alpha_{2}, \alpha_{3}\right), i=1,2$, are forms of degree four in the parameters $\alpha_{1}, \alpha_{2}, \alpha_{3}$. With these values of $\lambda$ and $\mu$, a solution of (III') may now be obtained using (4.7), and it may be written as

$$
\begin{aligned}
X_{1} & =\varrho\left\{L_{1}\left(\alpha_{1}, 0,-\alpha_{3}\right) F_{1}\left(\alpha_{j}\right)+L_{1}\left(\alpha_{1},-\alpha_{2}, 0\right) F_{2}\left(\alpha_{j}\right)\right\} \alpha_{1}, \\
X_{2} & =\varrho L_{1}\left(\alpha_{1},-\alpha_{2}, 0\right) F_{2}\left(\alpha_{j}\right) \alpha_{2}, \\
X_{3} & =\varrho\left\{L_{1}\left(\alpha_{1},-\alpha_{2}, 0\right)\left(F_{1}\left(\alpha_{j}\right)+F_{2}\left(\alpha_{j}\right)\right)\right\} \alpha_{3}, \\
Y_{1} & =\varrho\left\{L_{1}\left(\alpha_{1},-\alpha_{2}, 0\right)\left(F_{1}\left(\alpha_{j}\right)+F_{2}\left(\alpha_{j}\right)\right)\right\} \alpha_{1}, \\
Y_{2} & =\varrho\left\{L_{1}\left(\alpha_{1}, 0,-\alpha_{3}\right) F_{1}\left(\alpha_{j}\right)+L_{1}\left(\alpha_{1},-\alpha_{2}, 0\right) F_{2}\left(\alpha_{j}\right)\right\} \alpha_{2}, \\
Y_{3} & =\varrho L_{1}\left(\alpha_{1},-\alpha_{2}, 0\right) F_{2}\left(\alpha_{j}\right) \alpha_{3},
\end{aligned}
$$

where $\alpha_{1}, \alpha_{2}, \alpha_{3}$ are arbitrary non-zero integer parameters, while $\varrho$ is an arbitrary rational parameter.

We will now prove that (4.10) gives the complete solution of system (III') with $X_{1} X_{2} X_{3} \neq 0$ and $X_{3} \neq Y_{3}$. Let $X_{j}=\xi_{j}, Y_{j}=\eta_{j}, j=1,2,3$, be any arbitrary solution of (III') with $X_{1} X_{2} X_{3} \neq 0$ and $X_{3} \neq Y_{3}$ so that

$$
\begin{aligned}
L\left(\xi_{1}, \xi_{2}, \xi_{3}\right) & =L\left(\eta_{1}, \eta_{2}, \eta_{3}\right), \\
Q\left(\xi_{1}, \xi_{2}, \xi_{3}\right) & =Q\left(\eta_{1}, \eta_{2}, \eta_{3}\right), \\
\xi_{1} \xi_{2} \xi_{3} & =\eta_{1} \eta_{2} \eta_{3},
\end{aligned}
$$


and also $\xi_{j} \neq 0, \eta_{j} \neq 0, j=1,2,3$, and $\xi_{3}-\eta_{3} \neq 0$. We shall show that with suitably chosen values of the parameters $\alpha_{1}, \alpha_{2}, \alpha_{3}$ and $\varrho$, the solution (4.10) generates the arbitrarily chosen solution. We assign to $\alpha_{1}, \alpha_{2}, \alpha_{3}$ the values $\alpha_{1}^{\prime}, \alpha_{2}^{\prime}, \alpha_{3}^{\prime}$ respectively where

$$
\alpha_{1}^{\prime}=\eta_{1} \eta_{3}, \quad \alpha_{2}^{\prime}=\xi_{2} \xi_{3}, \quad \alpha_{3}^{\prime}=\xi_{3} \eta_{3},
$$

and we write

$$
\lambda_{1}=\frac{\xi_{1} \xi_{3}-\eta_{1} \eta_{3}}{\eta_{1}\left(\xi_{3}-\eta_{3}\right)}, \quad \mu_{1}=\frac{\xi_{3}-\eta_{3}}{\eta_{3}} .
$$

We now note that

$$
\begin{aligned}
& \left(\lambda_{1} \mu_{1}+1\right) \alpha_{1}^{\prime}=\xi_{1} \xi_{3}, \quad\left(\mu_{1}+1\right) \alpha_{1}^{\prime}=\xi_{3} \eta_{1}, \\
& \alpha_{2}^{\prime}=\xi_{2} \xi_{3}, \quad\left(\lambda_{1} \mu_{1}+1\right) \alpha_{2}^{\prime}=\xi_{3} \eta_{2}, \\
& \left(\mu_{1}+1\right) \alpha_{3}^{\prime}=\xi_{3}^{2}, \quad \alpha_{3}^{\prime}=\xi_{3} \eta_{3} .
\end{aligned}
$$

In view of (4.11) and (4.14), it follows that when we choose $\alpha_{j}=\alpha_{j}^{\prime}$, equations (4.8) and (4.9) are satisfied by taking $\lambda=\lambda_{1}$ and $\mu=\mu_{1}$. As was observed earlier, these equations have unique solutions which we had taken as $\lambda_{0}$ and $\mu_{0}$. It follows that $\lambda_{0}=\lambda_{1}$ and $\mu_{0}=\mu_{1}$ and so we have

$$
\begin{aligned}
& \lambda_{0}=\frac{L_{1}\left(\alpha_{1}^{\prime}, 0,-\alpha_{3}^{\prime}\right)}{L_{1}\left(\alpha_{1}^{\prime},-\alpha_{2}^{\prime}, 0\right)}=\frac{\xi_{1} \xi_{3}-\eta_{1} \eta_{3}}{\eta_{1}\left(\xi_{3}-\eta_{3}\right)}, \\
& \mu_{0}=\frac{F_{1}\left(\alpha_{1}^{\prime}, \alpha_{2}^{\prime}, \alpha_{3}^{\prime}\right)}{F_{2}\left(\alpha_{1}^{\prime}, \alpha_{2}^{\prime}, \alpha_{3}^{\prime}\right)}=\frac{\xi_{3}-\eta_{3}}{\eta_{3}},
\end{aligned}
$$

and hence there exist non-zero rational numbers $k_{1}$ and $k_{2}$ such that

$$
\begin{aligned}
L_{1}\left(\alpha_{1}^{\prime}, 0,-\alpha_{3}^{\prime}\right) & =k_{1}\left(\xi_{1} \xi_{3}-\eta_{1} \eta_{3}\right), \\
L_{1}\left(\alpha_{1}^{\prime},-\alpha_{2}^{\prime}, 0\right) & =k_{1} \eta_{1}\left(\xi_{3}-\eta_{3}\right), \\
F_{1}\left(\alpha_{1}^{\prime}, \alpha_{2}^{\prime}, \alpha_{3}^{\prime}\right) & =k_{2}\left(\xi_{3}-\eta_{3}\right), \\
F_{2}\left(\alpha_{1}^{\prime}, \alpha_{2}^{\prime}, \alpha_{3}^{\prime}\right) & =k_{2} \eta_{3} .
\end{aligned}
$$

Now substituting $\alpha_{j}=\alpha_{j}^{\prime}$ in (4.10), using the values of $L_{1}\left(\alpha_{1}^{\prime}, 0,-\alpha_{3}^{\prime}\right)$, $L_{1}\left(\alpha_{1}^{\prime},-\alpha_{2}^{\prime}, 0\right), F_{1}\left(\alpha_{j}^{\prime}\right), F_{2}\left(\alpha_{j}^{\prime}\right)$ given by (4.16), and taking

$$
\varrho=\frac{1}{k_{1} k_{2} \xi_{3} \eta_{1} \eta_{3}\left(\xi_{3}-\eta_{3}\right)},
$$

we find that the solution generated by (4.10) is precisely the given solution $X_{j}=\xi_{j}, Y_{j}=\eta_{j}, j=1,2,3$. This completes the proof that (4.10) gives all the solutions of system (III') with $X_{1} X_{2} X_{3} \neq 0$ and $X_{3} \neq Y_{3}$.

As an example, we consider the diophantine system

$$
\begin{aligned}
x_{1}+x_{2}+x_{3} & =y_{1}+y_{2}+y_{3}, \\
x_{1}^{2}+2 x_{2}^{2}+3 x_{3}^{2} & =y_{1}^{2}+2 y_{2}^{2}+3 y_{3}^{2}, \\
x_{1}^{3}+x_{2}^{3}+x_{3}^{3} & =y_{1}^{3}+y_{2}^{3}+y_{3}^{3} .
\end{aligned}
$$


To solve it, we replace (4.19) by

$$
\left(x_{1}+x_{2}+x_{3}\right)^{3}-\left(x_{1}^{3}+x_{2}^{3}+x_{3}^{3}\right)=\left(y_{1}+y_{2}+y_{3}\right)^{3}-\left(y_{1}^{3}+y_{2}^{3}+y_{3}^{3}\right),
$$

which reduces to

$$
\left(x_{1}+x_{2}\right)\left(x_{2}+x_{3}\right)\left(x_{3}+x_{1}\right)=\left(y_{1}+y_{2}\right)\left(y_{2}+y_{3}\right)\left(y_{3}+y_{1}\right) .
$$

To solve equations (4.17), (4.18) and (4.21), we make the invertible linear transformation

$$
\begin{array}{ll}
x_{1}=-X_{1}+X_{2}+X_{3}, & y_{1}=-Y_{1}+Y_{2}+Y_{3}, \\
x_{2}=X_{1}-X_{2}+X_{3}, & y_{2}=Y_{1}-Y_{2}+Y_{3}, \\
x_{3}=X_{1}+X_{2}-X_{3}, & y_{3}=Y_{1}+Y_{2}-Y_{3},
\end{array}
$$

so that (4.17) and (4.21) reduce to

$$
X_{1}+X_{2}+X_{3}=Y_{1}+Y_{2}+Y_{3}, \quad X_{1} X_{2} X_{3}=Y_{1} Y_{2} Y_{3},
$$

while (4.18) reduces to

$$
\begin{aligned}
3 X_{1}^{2}+3 X_{2}^{2}+3 X_{3}^{2}-2 X_{1} X_{3}-4 X_{2} X_{3} & \\
= & 3 Y_{1}^{2}+3 Y_{2}^{2}+3 Y_{3}^{2}-2 Y_{1} Y_{3}-4 Y_{2} Y_{3} .
\end{aligned}
$$

We can now obtain all solutions of (4.23) and (4.24) as described above, and hence obtain the complete solution of the system (4.17)-(4.19). All solutions of this system with both sides of (4.21) being 0 are readily determined. For instance, if $\alpha_{1}, \alpha_{2}$ and $\alpha_{3}$ are integers satisfying $3 \alpha_{1}^{2}+2 \alpha_{2}^{2}-5 \alpha_{3}^{2}=0$, then $\left(x_{1}, x_{2}, x_{3}, y_{1}, y_{2}, y_{3}\right)=\left(\alpha_{1},-\alpha_{1}, \alpha_{2}, \alpha_{2}, \alpha_{3},-\alpha_{3}\right)$ is a solution of the given diophantine system. All non-trivial solutions of the system (4.17)-(4.19) with both sides of (4.21) being non-zero are given by

$$
\begin{aligned}
x_{1}= & \varrho\left(3 \alpha_{1}^{3} \alpha_{2}^{2}-8 \alpha_{1}^{3} \alpha_{2} \alpha_{3}+4 \alpha_{1}^{3} \alpha_{3}^{2}-3 \alpha_{1}^{2} \alpha_{2}^{3}+9 \alpha_{1}^{2} \alpha_{2}^{2} \alpha_{3}\right. \\
& \left.-4 \alpha_{1}^{2} \alpha_{3}^{3}-11 \alpha_{1} \alpha_{2}^{2} \alpha_{3}^{2}+10 \alpha_{1} \alpha_{2} \alpha_{3}^{3}+5 \alpha_{2}^{3} \alpha_{3}^{2}-5 \alpha_{2}^{2} \alpha_{3}^{3}\right), \\
x_{2}= & \varrho\left(-3 \alpha_{1}^{3} \alpha_{2}^{2}+8 \alpha_{1}^{3} \alpha_{2} \alpha_{3}-4 \alpha_{1}^{3} \alpha_{3}^{2}+3 \alpha_{1}^{2} \alpha_{2}^{3}-3 \alpha_{1}^{2} \alpha_{2}^{2} \alpha_{3}\right. \\
& \left.-6 \alpha_{1}^{2} \alpha_{2} \alpha_{3}^{2}+4 \alpha_{1}^{2} \alpha_{3}^{3}-6 \alpha_{1} \alpha_{2}^{3} \alpha_{3}+7 \alpha_{1} \alpha_{2}^{2} \alpha_{3}^{2}+5 \alpha_{2}^{3} \alpha_{3}^{2}-5 \alpha_{2}^{2} \alpha_{3}^{3}\right), \\
x_{3}= & \varrho\left(3 \alpha_{1}^{3} \alpha_{2}^{2}-4 \alpha_{1}^{3} \alpha_{3}^{2}-3 \alpha_{1}^{2} \alpha_{2}^{3}-7 \alpha_{1}^{2} \alpha_{2}^{2} \alpha_{3}+8 \alpha_{1}^{2} \alpha_{2} \alpha_{3}^{2}\right. \\
& \left.+4 \alpha_{1}^{2} \alpha_{3}^{3}+6 \alpha_{1} \alpha_{2}^{3} \alpha_{3}+3 \alpha_{1} \alpha_{2}^{2} \alpha_{3}^{2}-10 \alpha_{1} \alpha_{2} \alpha_{3}^{3}-5 \alpha_{2}^{3} \alpha_{3}^{2}+5 \alpha_{2}^{2} \alpha_{3}^{3}\right), \\
y_{1}= & \varrho\left(-3 \alpha_{1}^{3} \alpha_{2}^{2}+6 \alpha_{1}^{3} \alpha_{2} \alpha_{3}-4 \alpha_{1}^{3} \alpha_{3}^{2}+3 \alpha_{1}^{2} \alpha_{2}^{3}+3 \alpha_{1}^{2} \alpha_{2}^{2} \alpha_{3}\right. \\
& \left.-8 \alpha_{1}^{2} \alpha_{2} \alpha_{3}^{2}+4 \alpha_{1}^{2} \alpha_{3}^{3}-10 \alpha_{1} \alpha_{2}^{3} \alpha_{3}+9 \alpha_{1} \alpha_{2}^{2} \alpha_{3}^{2}+5 \alpha_{2}^{3} \alpha_{3}^{2}-5 \alpha_{2}^{2} \alpha_{3}^{3}\right), \\
y_{2}= & \varrho\left(3 \alpha_{1}^{3} \alpha_{2}^{2}-4 \alpha_{1}^{3} \alpha_{3}^{2}-3 \alpha_{1}^{2} \alpha_{2}^{3}-9 \alpha_{1}^{2} \alpha_{2}^{2} \alpha_{3}+10 \alpha_{1}^{2} \alpha_{2} \alpha_{3}^{2}\right. \\
& \left.+4 \alpha_{1}^{2} \alpha_{3}^{3}+10 \alpha_{1} \alpha_{2}^{3} \alpha_{3}-3 \alpha_{1} \alpha_{2}^{2} \alpha_{3}^{2}-8 \alpha_{1} \alpha_{2} \alpha_{3}^{3}-5 \alpha_{2}^{3} \alpha_{3}^{2}+5 \alpha_{2}^{2} \alpha_{3}^{3}\right), \\
y_{3}= & \varrho\left(3 \alpha_{1}^{3} \alpha_{2}^{2}-6 \alpha_{1}^{3} \alpha_{2} \alpha_{3}+4 \alpha_{1}^{3} \alpha_{3}^{2}-3 \alpha_{1}^{2} \alpha_{2}^{3}+5 \alpha_{1}^{2} \alpha_{2}^{2} \alpha_{3}\right. \\
& \left.-4 \alpha_{1}^{2} \alpha_{3}^{3}-7 \alpha_{1} \alpha_{2}^{2} \alpha_{3}^{2}+8 \alpha_{1} \alpha_{2} \alpha_{3}^{3}+5 \alpha_{2}^{3} \alpha_{3}^{2}-5 \alpha_{2}^{2} \alpha_{3}^{3}\right),
\end{aligned}
$$

where $\alpha_{1}, \alpha_{2}$ and $\alpha_{3}$ are arbitrary parameters while $\varrho$ is an arbitrary rational number. 
5. The diophantine system (IV). We now consider the system (IV), that is,

$$
\begin{aligned}
L\left(x_{1}, x_{2}, x_{3}\right) & =L\left(y_{1}, y_{2}, y_{3}\right), \\
C_{1}\left(x_{1}, x_{2}, x_{3}\right) & =C_{1}\left(y_{1}, y_{2}, y_{3}\right), \\
C_{2}\left(x_{1}, x_{2}, x_{3}\right) & =C_{2}\left(y_{1}, y_{2}, y_{3}\right) .
\end{aligned}
$$

A special case of this system was solved in [1, pp. 301-302]. To solve the general system (IV), we will follow the same procedures as in the case of (I) and just make the required changes to take into account the additional linear equation. Thus given a non-trivial solution $x_{j}=\xi_{j}, y_{j}=\eta_{j}, j=1,2,3$, of (IV), we use the substitutions (2.4), and proceeding as before, obtain the cubic curve (2.6). We note that the values of $x_{j}, y_{j}, j=1,2,3$, given by (2.4) satisfy equation (5.1) for arbitrary values of $X_{1}, X_{2}$ and $X_{3}$ since $L\left(\xi_{1}, \xi_{2}, \xi_{3}\right)=L\left(\eta_{1}, \eta_{2}, \eta_{3}\right)$, and hence, as in the case of (I), we can, in general, find new solutions of (IV) by determining rational points on the cubic curve (2.6).

Further, as in the case of (I), for the existence of non-trivial solutions of (IV) with $x_{1} \neq y_{1}$ and $x_{1} y_{2}-x_{2} y_{1} \neq 0$, a necessary and sufficient condition of the type (2.12) may be obtained where, in view of the additional equation (5.1), the parameters $a_{1}, a_{2}, a_{3}$ must be chosen to satisfy the linear condition $L\left(a_{1}, a_{2}, a_{3}\right)=0$.

As a specific example, we consider the diophantine system

$$
\begin{aligned}
x_{1}+x_{2}+x_{3} & =y_{1}+y_{2}+y_{3}, \\
x_{1}^{3}+2 x_{2}^{3}+3 x_{3}^{3} & =y_{1}^{3}+2 y_{2}^{3}+3 y_{3}^{3}, \\
x_{1}^{3}+x_{2}^{3}+x_{3}^{3} & =y_{1}^{3}+y_{2}^{3}+y_{3}^{3} .
\end{aligned}
$$

A necessary and sufficient condition for the solvability of this system, obtained as above, is that there exist integers $a_{1}$ and $a_{2}$ such that the equation

$$
\begin{aligned}
\sigma^{2}= & -3 a_{2}^{2}\left(2 a_{1}+a_{2}\right)^{2}\left(a_{1}^{2}+a_{1} a_{2}+a_{2}^{2}\right)^{2} \varrho^{4} \\
& -12 a_{2}^{3}\left(2 a_{1}+a_{2}\right)\left(a_{1}^{2}+a_{1} a_{2}+a_{2}^{2}\right)\left(a_{1}+a_{2}\right)^{2} \varrho^{3} \\
& -18 a_{2}^{2}\left(a_{1}+a_{2}\right)\left(2 a_{1}^{5}+2 a_{1}^{4} a_{2}+2 a_{1}^{3} a_{2}^{2}+3 a_{1}^{2} a_{2}^{3}+3 a_{1} a_{2}^{4}+a_{2}^{5}\right) \varrho^{2} \\
& -12 a_{2}^{2}\left(2 a_{1}^{3}+a_{2}^{3}\right)\left(a_{1}+a_{2}\right)^{3} \varrho-3\left(a_{1}+a_{2}\right)^{2}\left(2 a_{1}^{3}+a_{2}^{3}\right)^{2},
\end{aligned}
$$

is satisfied by rational values of $\varrho$ and $\sigma$. Taking $a_{1}=1, a_{2}=-2$, equation (5.5) reduces to a quadratic equation whose parametric solution leads to the following parametric solution of the system (5.4):

$$
\begin{array}{ll}
x_{1}=-\alpha_{1}^{2}+4 \alpha_{1} \alpha_{2}-4 \alpha_{2}^{2}, & y_{1}=-\alpha_{1}^{2}-4 \alpha_{1} \alpha_{2}-4 \alpha_{2}^{2}, \\
x_{2}=-\alpha_{1}^{2}-8 \alpha_{1} \alpha_{2}+4 \alpha_{2}^{2}, & y_{2}=-\alpha_{1}^{2}+8 \alpha_{1} \alpha_{2}+4 \alpha_{2}^{2}, \\
x_{3}=\alpha_{1}^{2}+4 \alpha_{1} \alpha_{2}+4 \alpha_{2}^{2}, & y_{3}=\alpha_{1}^{2}-4 \alpha_{1} \alpha_{2}+4 \alpha_{2}^{2} .
\end{array}
$$

This is not the complete solution of (5.4). To get more solutions, it was 
found by trial that when we take $a_{1}=7, a_{2}=-8$, equation (5.5) represents an elliptic curve of rank 3 , and the infinitely many rational points on this curve yield infinitely many numerical solutions of (5.4), one such solution being $\left(x_{1}, x_{2}, x_{3}, y_{1}, y_{2}, y_{3}\right)=(168,-83,242,-98,221,204)$.

6. The diophantine system (V). We now consider the system (V), that is,

$$
\begin{aligned}
& Q_{1}\left(x_{1}, x_{2}, x_{3}\right)=Q_{1}\left(y_{1}, y_{2}, y_{3}\right), \\
& Q_{2}\left(x_{1}, x_{2}, x_{3}\right)=Q_{2}\left(y_{1}, y_{2}, y_{3}\right), \\
& Q_{3}\left(x_{1}, x_{2}, x_{3}\right)=Q_{3}\left(y_{1}, y_{2}, y_{3}\right) .
\end{aligned}
$$

We write

$$
x_{j}=X_{j}+Y_{j}, \quad y_{j}=X_{j}-Y_{j}, \quad j=1,2,3,
$$

so that the given diophantine system may be replaced by

$$
\begin{aligned}
& L_{11}\left(X_{1}, X_{2}, X_{3}\right) Y_{1}+L_{12}\left(X_{1}, X_{2}, X_{3}\right) Y_{2}+L_{13}\left(X_{1}, X_{2}, X_{3}\right) Y_{3}=0, \\
& L_{21}\left(X_{1}, X_{2}, X_{3}\right) Y_{1}+L_{22}\left(X_{1}, X_{2}, X_{3}\right) Y_{2}+L_{23}\left(X_{1}, X_{2}, X_{3}\right) Y_{3}=0, \\
& L_{31}\left(X_{1}, X_{2}, X_{3}\right) Y_{1}+L_{32}\left(X_{1}, X_{2}, X_{3}\right) Y_{2}+L_{33}\left(X_{1}, X_{2}, X_{3}\right) Y_{3}=0,
\end{aligned}
$$

where the forms $L_{i j}\left(X_{1}, X_{2}, X_{3}\right), i=1,2,3, j=1,2,3$, are given by

$$
L_{i j}\left(X_{1}, X_{2}, X_{3}\right)=\left(\frac{\partial Q_{i}}{\partial x_{j}}\right)_{x_{1}=X_{1}, x_{2}=X_{2}, x_{3}=X_{3}} .
$$

The equations (6.5) may be considered as three linear equations in the variables $Y_{1}, Y_{2}$, and $Y_{3}$ and they will have a non-zero solution if and only if

$$
\left|\begin{array}{lll}
L_{11}\left(X_{1}, X_{2}, X_{3}\right) & L_{12}\left(X_{1}, X_{2}, X_{3}\right) & L_{13}\left(X_{1}, X_{2}, X_{3}\right) \\
L_{21}\left(X_{1}, X_{2}, X_{3}\right) & L_{22}\left(X_{1}, X_{2}, X_{3}\right) & L_{23}\left(X_{1}, X_{2}, X_{3}\right) \\
L_{31}\left(X_{1}, X_{2}, X_{3}\right) & L_{32}\left(X_{1}, X_{2}, X_{3}\right) & L_{33}\left(X_{1}, X_{2}, X_{3}\right)
\end{array}\right|=0 .
$$

Equation (6.7) represents a cubic curve in the projective plane and any rational point $\left(X_{1}, X_{2}, X_{3}\right)$ on this curve would lead, on solving the equations (6.5), and using the relations (6.4), to a solution of the given diophantine system. In general, the cubic curve (6.7) is of genus 1 and it may have infinitely many or a finite number (possibly zero) of rational points. We also note that no algorithm is known that will always find a rational point on an arbitrary curve of genus 1 . If in a particular case, the cubic equation (6.7) represents a curve of genus 0 , its complete solution can be determined, and this leads, as indicated, to a complete solution of the given diophantine system. If, however, (6.7) represents a curve of genus 1, even though there is no algorithm that works in all cases, we may still search for rational points on the curve, for instance by using a computer program such as APECS, and obtain either all the finitely many rational points on the curve (6.7), or, 
if (6.7) represents an elliptic curve of positive rank, we may possibly obtain infinitely many rational points on it. These rational points $\left(X_{1}, X_{2}, X_{3}\right)$ can then be used to obtain solutions of the given diophantine system.

Finally, we note that when one of the quadratic forms $Q_{i}\left(x_{j}\right)$, say $Q_{1}\left(x_{j}\right)$, has only two variables, say, $x_{1}$ and $x_{2}$, it is easily seen that the forms $L_{1 j}\left(X_{1}, X_{2}, X_{3}\right), j=1,2,3$, are independent of $X_{3}$, and hence $(0,0,1)$ is a rational point on the curve (6.7). In general, this rational point may be used to find infinitely many points on the cubic curve (6.7) by using the tangent and chord method, and we thus obtain infinitely many solutions of $(\mathrm{V})$.

7. The diophantine system (VI). We now consider the system (VI), that is,

$$
\begin{aligned}
Q_{1}\left(x_{1}, x_{2}, x_{3}\right) & =Q_{1}\left(y_{1}, y_{2}, y_{3}\right), \\
Q_{2}\left(x_{1}, x_{2}, x_{3}\right) & =Q_{2}\left(y_{1}, y_{2}, y_{3}\right), \\
C\left(x_{1}, x_{2}, x_{3}\right) & =C\left(y_{1}, y_{2}, y_{3}\right) .
\end{aligned}
$$

We will first show that if a non-trivial solution of this system is known, we can, in general, use it to find another solution. If $x_{j}=\xi_{j}, y_{j}=\eta_{j}$, $j=1,2,3$, is the known non-trivial solution, we have $Q_{1}\left(\xi_{j}\right)=Q_{1}\left(\eta_{j}\right)$, $Q_{2}\left(\xi_{j}\right)=Q_{2}\left(\eta_{j}\right)$, and $C\left(\xi_{j}\right)=C\left(\eta_{j}\right)$. In (7.1)-(7.3), we now substitute

$$
x_{j}=a_{j} \theta+\xi_{j}, \quad y_{j}=a_{j} \theta+\eta_{j}, \quad j=1,2,3,
$$

where $a_{1}, a_{2}, a_{3}$ and $\theta$ are arbitrary parameters with $\theta \neq 0$. In each of these equations, we transpose all the terms to one side and remove the factor $\theta$, so that equations (7.1)-(7.3) now reduce respectively to the following equations:

$$
\begin{aligned}
& \sum_{j=1}^{3}\left\{\left(\frac{\partial Q_{1}}{\partial x_{j}}\right)_{x_{j}=\xi_{j}}-\left(\frac{\partial Q_{1}}{\partial x_{j}}\right)_{x_{j}=\eta_{j}}\right\} a_{j}=0, \\
& \sum_{j=1}^{3}\left\{\left(\frac{\partial Q_{2}}{\partial x_{j}}\right)_{x_{j}=\xi_{j}}-\left(\frac{\partial Q_{2}}{\partial x_{j}}\right)_{x_{j}=\eta_{j}}\right\} a_{j}=0,
\end{aligned}
$$

and

$$
\begin{aligned}
\frac{\theta}{2}\left[\left\{\sum_{j=1}^{3}\left(a_{j} \frac{\partial}{\partial x_{j}}\right)^{2} C\right\}_{x_{j}=\xi_{j}}-\left\{\sum_{j=1}^{3}\left(a_{j} \frac{\partial}{\partial x_{j}}\right)^{2} C\right\}_{x_{j}=\eta_{j}}\right] \\
+\left\{\sum_{j=1}^{3}\left(a_{j} \frac{\partial C}{\partial x_{j}}\right)\right\}_{x_{j}=\xi_{j}}-\left\{\sum_{j=1}^{3}\left(a_{j} \frac{\partial C}{\partial x_{j}}\right)\right\}_{x_{j}=\eta_{j}}=0 .
\end{aligned}
$$

We solve the linear equations (7.5) and (7.6) to obtain a non-zero solution for $a_{1}, a_{2}$ and $a_{3}$, and with these values of $a_{1}, a_{2}$ and $a_{3}$, we may, in general, 
solve equation (7.7) to get a non-zero solution for $\theta$, and thus obtain a new solution of (VI) using (7.4). There are certain solutions of (VI) that do not lead to a new solution using the above procedure. These include solutions of the type $x_{j}=\alpha_{j}, y_{j}=-\alpha_{j}, j=1,2,3$, where $C\left(\alpha_{1}, \alpha_{2}, \alpha_{3}\right)=0$. Such solutions are found by simply solving the single diophantine equation $C\left(x_{1}, x_{2}, x_{3}\right)=0$, and, strictly speaking, cannot be regarded as non-trivial solutions of the system (VI).

We will now obtain a necessary and sufficient condition for the solvability of (VI). In (7.1)-(7.3), we substitute

$$
y_{j}=x_{j}+a_{j} m, \quad j=1,2,3,
$$

where $a_{1}, a_{2}, a_{3}$ and $m$ are arbitrary parameters with $m \neq 0$. Now on transposing all the terms of (7.1) and (7.2) to one side, and removing the factor $m$, we get two homogeneous linear equations in $x_{1}, x_{2}, x_{3}$ and $m$, and similarly (7.3) reduces to a homogeneous quadratic equation in $x_{1}, x_{2}, x_{3}$ and $m$. Eliminating $x_{2}$ and $x_{3}$ from these three equations, we get a quadratic equation in $x_{1}$ and $m$ which may be written as $Q\left(x_{1}, m\right)=0$, where

$$
Q\left(x_{1}, m\right)=\phi_{0}\left(a_{1}, a_{2}, a_{3}\right) x_{1}^{2}+\phi_{1}\left(a_{1}, a_{2}, a_{3}\right) x_{1} m+\phi_{2}\left(a_{1}, a_{2}, a_{3}\right) m^{2},
$$

and $\phi_{j}\left(a_{1}, a_{2}, a_{3}\right), j=0,1,2$, are forms of degree 5,6 and 7 respectively in the parameters $a_{1}, a_{2}$ and $a_{3}$. A necessary and sufficient condition for the solvability of the quadratic equation $Q\left(x_{1}, m\right)=0$, and hence for the solvability of the diophantine system (VI), is that the discriminant of the quadratic form $Q\left(x_{1}, m\right)$ is a perfect square. This discriminant has the square of a quadratic form in $a_{1}, a_{2}, a_{3}$ as a factor, and so this factor may be removed. Thus a necessary and sufficient condition for the solvability of (VI) is that there exist integers $a_{1}, a_{2}, a_{3}$ and $\varrho$ such that

$$
\phi\left(a_{1}, a_{2}, a_{3}\right)=\varrho^{2},
$$

where $\phi\left(a_{1}, a_{2}, a_{3}\right)$, a form of degree eight in $a_{1}, a_{2}, a_{3}$, is obtained by removing the squared factor from the discriminant of $Q\left(x_{1}, m\right)$.

As a specific example, we consider the diophantine system

$$
\begin{aligned}
x_{1}^{2}+x_{2}^{2} & =y_{1}^{2}+y_{2}^{2}, \\
x_{1}^{2}+x_{3}^{2} & =y_{1}^{2}+y_{3}^{2}, \\
x_{1}^{3}+x_{2}^{3}+x_{3}^{3} & =y_{1}^{3}+y_{2}^{3}+y_{3}^{3} .
\end{aligned}
$$

A necessary and sufficient condition for the solvability of this system, obtained as above, is given by

$$
-3 a_{1} a_{2} a_{3}\left(a_{1} a_{2}+a_{2} a_{3}+a_{3} a_{1}\right)\left(a_{1}^{3}+a_{2}^{3}+a_{3}^{3}\right)=\varrho^{2},
$$

which, on substituting $a_{3}=-a_{1}-a_{2}$, reduces to

$$
9 a_{1}^{2} a_{2}^{2}\left(a_{1}+a_{2}\right)^{2}\left(a_{1}^{2}+a_{1} a_{2}+a_{2}^{2}\right)=\varrho^{2},
$$


and taking

$$
a_{1}=-\alpha_{1}^{2}-2 \alpha_{1} \alpha_{2}, \quad a_{2}=\alpha_{1}^{2}-\alpha_{2}^{2}, \quad a_{3}=2 \alpha_{1} \alpha_{2}+\alpha_{2}^{2},
$$

the condition (7.12) is satisfied, which leads to the parametric solution

$$
\begin{aligned}
& x_{1}=\alpha_{1}^{4}+\alpha_{1}^{3} \alpha_{2}+2 \alpha_{1}^{2} \alpha_{2}^{2}+4 \alpha_{1} \alpha_{2}^{3}+\alpha_{2}^{4}, \\
& x_{2}=-\alpha_{1}^{4}-3 \alpha_{1}^{3} \alpha_{2}-5 \alpha_{1}^{2} \alpha_{2}^{2}-\alpha_{1} \alpha_{2}^{3}+\alpha_{2}^{4}, \\
& x_{3}=-\alpha_{1}^{4}-4 \alpha_{1}^{3} \alpha_{2}-2 \alpha_{1}^{2} \alpha_{2}^{2}-\alpha_{1} \alpha_{2}^{3}-\alpha_{2}^{4}, \\
& y_{1}=-\alpha_{1}^{4}-5 \alpha_{1}^{3} \alpha_{2}-4 \alpha_{1}^{2} \alpha_{2}^{2}+\alpha_{2}^{4}, \\
& y_{2}=\alpha_{1}^{4}-\alpha_{1}^{3} \alpha_{2}-5 \alpha_{1}^{2} \alpha_{2}^{2}-3 \alpha_{1} \alpha_{2}^{3}-\alpha_{2}^{4}, \\
& y_{3}=-\alpha_{1}^{4}+4 \alpha_{1}^{2} \alpha_{2}^{2}+5 \alpha_{1} \alpha_{2}^{3}+\alpha_{2}^{4},
\end{aligned}
$$

of the diophantine system (7.11).

8. The diophantine system (VII). We now consider the system (VII), that is,

$$
\begin{aligned}
Q\left(x_{1}, x_{2}, x_{3}\right) & =Q\left(y_{1}, y_{2}, y_{3}\right), \\
C_{1}\left(x_{1}, x_{2}, x_{3}\right) & =C_{1}\left(y_{1}, y_{2}, y_{3}\right), \\
C_{2}\left(x_{1}, x_{2}, x_{3}\right) & =C_{2}\left(y_{1}, y_{2}, y_{3}\right) .
\end{aligned}
$$

As in the case of $(\mathrm{I})$, we may assume without loss that for a non-trivial solution of this system, $x_{1} \neq y_{1}$ and $x_{1} y_{2}-x_{2} y_{1} \neq 0$. To solve this system, we use the substitutions (2.7) so that equation (8.1) reduces to

$$
2 Q\left(a_{1}, a_{2}, a_{3}\right) m X_{2}+\left\{\left(X_{2} \frac{\partial}{\partial x_{2}}+X_{3} \frac{\partial}{\partial x_{3}}\right)\left(\sum_{j=1}^{3} a_{j} \frac{\partial}{\partial x_{j}}\right) Q\right\}=0,
$$

while equations (8.2) and (8.3) yield the two equations (2.8). Eliminating $m$ and $X_{3}$ from (2.8) and (8.4), we obtain an equation that may be written as

$$
\phi_{0}\left(a_{1}, a_{2}, a_{3}\right) X_{1}^{4}+\phi_{1}\left(a_{1}, a_{2}, a_{3}\right) X_{1}^{2} X_{2}^{2}+\phi_{2}\left(a_{1}, a_{2}, a_{3}\right) X_{2}^{4}=0,
$$

where $\phi_{j}\left(a_{1}, a_{2}, a_{3}\right), j=0,1,2$, are forms of degrees 28, 26 and 24 respectively in the parameters $a_{1}, a_{2}, a_{3}$. If for certain rational values of $a_{1}, a_{2}, a_{3}$, equation (8.5) has a rational non-zero solution for $X_{1}, X_{2}$, we can solve (2.8) and (8.4) to obtain rational solutions for $m$ and $X_{3}$, and hence obtain a non-trivial solution of (VII). Conversely, if there exists a solution $x_{j}=\xi_{j}, y_{j}=\eta_{j}, j=1,2,3$, of (VII) such that $\xi_{1} \neq \eta_{1}, \xi_{1} \eta_{2}-\xi_{2} \eta_{1} \neq 0$, it is easily seen, as in the case of (I), that when the values of the parameters $a_{1}, a_{2}, a_{3}, X_{1}, X_{2}, X_{3}$ and $m$ are chosen as in (2.11), equations (2.8) and (8.4) are satisfied, and hence (8.5) must also be satisfied by these values of the parameters. A necessary and sufficient condition for the solvability of (8.5), and hence of (VII), is that there exist integers $a_{1}, a_{2}, a_{3}$ such that either of the following two conditions is satisfied: 
(i) $\phi_{0}\left(a_{j}\right)=0$ and $-\phi_{1}\left(a_{j}\right) \phi_{2}\left(a_{j}\right)$ is a non-zero perfect square;

(ii) $\phi_{1}\left(a_{j}\right)^{2}-4 \phi_{0}\left(a_{j}\right) \phi_{2}\left(a_{j}\right)$ is a perfect square and one of the two values of $2 \phi_{0}\left(a_{j}\right)\left[-\phi_{1}\left(a_{j}\right) \pm\left\{\phi_{1}^{2}\left(a_{j}\right)-4 \phi_{0}\left(a_{j}\right) \phi_{2}\left(a_{j}\right)\right\}^{1 / 2}\right]$ is a non-zero perfect square.

In any given case, a computer program may easily be written to search for integers $a_{1}, a_{2}, a_{3}$ satisfying one or the other of the conditions given above.

As a specific example, we consider the system

$$
\begin{aligned}
x_{1}^{2}+2 x_{2}^{2}+x_{3}^{2} & =y_{1}^{2}+2 y_{2}^{2}+y_{3}^{2}, \\
x_{1}^{3}+x_{2}^{3}+x_{3}^{3} & =y_{1}^{3}+y_{2}^{3}+y_{3}^{3}, \\
x_{1} x_{2} x_{3} & =y_{1} y_{2} y_{3} .
\end{aligned}
$$

We obtain equation (8.5) for this system, and note that taking $a_{3}=-a_{1}-a_{2}$, the left-hand side of this equation has two linear factors, which equated to zero lead to trivial solutions of (8.6), and hence removing these factors, the condition (8.5) reduces in this case to

$$
\left(a_{1}+a_{2}\right)\left(2 a_{1}^{2}+2 a_{1} a_{2}+3 a_{2}^{2}\right)^{2} X_{1}^{2}-\left(13 a_{1}^{2}+13 a_{1} a_{2}-3 a_{2}^{2}\right) a_{1} X_{2}^{2}=0 .
$$

Now on writing

$$
a_{2}=-a_{1} \varrho / 3, \quad X_{2}=\left(-\varrho^{3}+5 \varrho^{2}-12 \varrho+18\right) a_{1} X_{1} /(3 \sigma),
$$

equation (8.7) reduces to

$$
\sigma^{2}=\varrho^{3}+10 \varrho^{2}-78 \varrho+117,
$$

which represents an elliptic curve of rank 1, a point of infinite order being $(\varrho, \sigma)=(-6,27)$. We can thus find infinitely many rational points on the curve (8.9), and hence obtain infinitely many solutions of the system (8.6), two such solutions being

$$
\begin{aligned}
& \left(x_{1}, x_{2}, x_{3}, y_{1}, y_{2}, y_{3}\right)=(5,1,-6,2,-5,3) \\
& \left(x_{1}, x_{2}, x_{3}, y_{1}, y_{2}, y_{3}\right)=(-2871,2491,380,423,-2755,2332) .
\end{aligned}
$$

All such solutions will satisfy the additional relation

$$
x_{1}+x_{2}+x_{3}=y_{1}+y_{2}+y_{3},
$$

since we had taken $a_{3}=-a_{1}-a_{2}$ in (8.5). To obtain solutions of (8.6) such that (8.10) does not hold, we must find solutions of (8.5) by trial. One such solution with $\left(a_{1}, a_{2}, a_{3}\right)=(7,6,-4)$ is $\left(x_{1}, x_{2}, x_{3}, y_{1}, y_{2}, y_{3}\right)=$ $(10,20,9,-18,-4,25)$. 
9. The diophantine system (VIII). Finally, we consider the system (VIII), that is,

$$
\begin{aligned}
& C_{1}\left(x_{1}, x_{2}, x_{3}\right)=C_{1}\left(y_{1}, y_{2}, y_{3}\right), \\
& C_{2}\left(x_{1}, x_{2}, x_{3}\right)=C_{2}\left(y_{1}, y_{2}, y_{3}\right), \\
& C_{3}\left(x_{1}, x_{2}, x_{3}\right)=C_{3}\left(y_{1}, y_{2}, y_{3}\right) .
\end{aligned}
$$

As in the case of (I), we assume without loss that $x_{1} \neq y_{1}$ and $x_{1} y_{2}-x_{2} y_{1}$ $\neq 0$, and by writing

$$
x_{3}=p x_{1}+q x_{2}, \quad y_{3}=p y_{1}+q y_{2},
$$

we reduce (VIII) to the following equivalent system $\left(\mathrm{VIII}^{\prime}\right)$ :

$$
\begin{aligned}
& C_{1}^{\prime}\left(x_{1}, x_{2}\right)=C_{1}^{\prime}\left(y_{1}, y_{2}\right), \\
& C_{2}^{\prime}\left(x_{1}, x_{2}\right)=C_{2}^{\prime}\left(y_{1}, y_{2}\right), \\
& C_{3}^{\prime}\left(x_{1}, x_{2}\right)=C_{3}^{\prime}\left(y_{1}, y_{2}\right),
\end{aligned}
$$

where the coefficients in the binary cubic forms $C_{i}^{\prime}\left(x_{1}, x_{2}\right)$ are polynomials in $p$ and $q$. It is easy to determine whether there exist rational values of $p$ and $q$ such that the forms $C_{1}^{\prime}\left(x_{1}, x_{2}\right), C_{2}^{\prime}\left(x_{1}, x_{2}\right)$ and $C_{3}^{\prime}\left(x_{1}, x_{2}\right)$ are linearly dependent. If such values can be determined, the diophantine system reduces to just two simultaneous symmetric cubic equations of ( $\left.\mathrm{VIII}^{\prime}\right)$, and a necessary and sufficient condition for the solvability of such a system has been obtained in [3, pp. 94-95]. A noteworthy example of such a reduction of (VIII) to two cubic equations arises for the system

$$
\begin{aligned}
C_{1}\left(x_{1}, x_{2}, x_{3}\right) & =C_{1}\left(y_{1}, y_{2}, y_{3}\right), \\
x_{1}^{3}+x_{2}^{3}+x_{3}^{3} & =y_{1}^{3}+y_{2}^{3}+y_{3}^{3}, \\
x_{1} x_{2} x_{3} & =y_{1} y_{2} y_{3},
\end{aligned}
$$

in which case the substitutions $x_{3}=-x_{1}-x_{2}, y_{3}=-y_{1}-y_{2}$ reduce both (9.9) and (9.10) to the equation $x_{1} x_{2}\left(x_{1}+x_{2}\right)=y_{1} y_{2}\left(y_{1}+y_{2}\right)$ and solutions of the system may be found as described in [3].

We will now obtain a necessary and sufficient condition for the solvability of $\left(\mathrm{VIII}^{\prime}\right)$ when the forms $C_{i}^{\prime}\left(x_{1}, x_{2}\right)$ are linearly independent. It follows from (9.5) and (9.6) that, for any $t$,

$$
\begin{aligned}
C_{2}^{\prime}(t, 1) C_{1}^{\prime}\left(x_{1}, x_{2}\right)-C_{1}^{\prime}(t, 1) C_{2}^{\prime}\left(x_{1}, x_{2}\right) \\
=C_{2}^{\prime}(t, 1) C_{1}^{\prime}\left(y_{1}, y_{2}\right)-C_{1}^{\prime}(t, 1) C_{2}^{\prime}\left(y_{1}, y_{2}\right),
\end{aligned}
$$

and similarly from (9.5) and (9.7), we derive

$$
\begin{aligned}
C_{3}^{\prime}(t, 1) C_{1}^{\prime}\left(x_{1}, x_{2}\right)-C_{1}^{\prime}(t, 1) C_{3}^{\prime}\left(x_{1}, x_{2}\right) & \\
& =C_{3}^{\prime}(t, 1) C_{1}^{\prime}\left(y_{1}, y_{2}\right)-C_{1}^{\prime}(t, 1) C_{3}^{\prime}\left(y_{1}, y_{2}\right) .
\end{aligned}
$$


It is also easily seen that when the two equations (9.11) and (9.12) have a solution that is valid for all values of $t$, then this solution also satisfies the system $\left(\mathrm{VIII}^{\prime}\right)$. Thus, we have reduced $\left(\mathrm{VIII}^{\prime}\right)$ to the system of equations (9.11) and (9.12) which must have a solution valid for all values of $t$.

It follows from (9.11) and (9.12) that, for all values of $t$,

$$
\begin{aligned}
\left\{C _ { 2 } ^ { \prime } ( t , 1 ) C _ { 1 } ^ { \prime } \left(x_{1},\right.\right. & \left.\left.x_{2}\right)-C_{1}^{\prime}(t, 1) C_{2}^{\prime}\left(x_{1}, x_{2}\right)\right\} \\
& \times\left\{C_{3}^{\prime}(t, 1) C_{1}^{\prime}\left(y_{1}, y_{2}\right)-C_{1}^{\prime}(t, 1) C_{3}^{\prime}\left(y_{1}, y_{2}\right)\right\} \\
& -\left\{C_{3}^{\prime}(t, 1) C_{1}^{\prime}\left(x_{1}, x_{2}\right)-C_{1}^{\prime}(t, 1) C_{3}^{\prime}\left(x_{1}, x_{2}\right)\right\} \\
& \times\left\{C_{2}^{\prime}(t, 1) C_{1}^{\prime}\left(y_{1}, y_{2}\right)-C_{1}^{\prime}(t, 1) C_{2}^{\prime}\left(y_{1}, y_{2}\right)\right\}=0 .
\end{aligned}
$$

It is easily seen that the left-hand side of (9.13) has the factors $x_{1} y_{2}-$ $x_{2} y_{1}, x_{1}-t x_{2}, y_{1}-t y_{2}$, and $C_{1}^{\prime}(t, 1)$. Since $x_{1} y_{2}-x_{2} y_{1} \neq 0$ and none of the other three factors can be 0 for all values of $t$, we may remove these factors from the left-hand side of (9.13) to get an equation which may be written as follows:

$$
t\left\{y_{1} L_{1}\left(x_{1}, x_{2}\right)+y_{2} L_{2}\left(x_{1}, x_{2}\right)\right\}+y_{1} L_{3}\left(x_{1}, x_{2}\right)+y_{2} L_{4}\left(x_{1}, x_{2}\right)=0,
$$

where $L_{i}\left(x_{1}, x_{2}\right), i=1,2,3,4$, are linear forms in $x_{1}$ and $x_{2}$ whose coefficients are functions of $p$ and $q$. Since (9.14) must be satisfied by any solution of ( $\mathrm{VIII}^{\prime}$ ) for all values of $t$, it follows that the following two equations must be simultaneously satisfied by any solution of $\left(\mathrm{VIII}^{\prime}\right)$ :

$$
\begin{aligned}
& y_{1} L_{1}\left(x_{1}, x_{2}\right)+y_{2} L_{2}\left(x_{1}, x_{2}\right)=0, \\
& y_{1} L_{3}\left(x_{1}, x_{2}\right)+y_{2} L_{4}\left(x_{1}, x_{2}\right)=0 .
\end{aligned}
$$

Eliminating $y_{1}$ and $y_{2}$ from (9.15), we get an equation

$$
Q\left(x_{1}, x_{2}\right)=0,
$$

where $Q\left(x_{1}, x_{2}\right)$ is a binary quadratic form with coefficients that are functions of $p$ and $q$. It follows that a necessary condition for the solvability of (VIII') is that the discriminant $\phi(p, q)$ of the quadratic form $Q\left(x_{1}, x_{2}\right)$ must be a perfect square. If there exist rational numbers $p$ and $q$ such that $\phi(p, q)$ is a perfect square, then with these values of $p$ and $q$, the complete solution of the equations (9.15) is readily determined and may be written as

$$
x_{1}=m_{1} \xi_{1}, \quad x_{2}=m_{1} \xi_{2}, \quad y_{1}=m_{2} \eta_{1}, \quad y_{2}=m_{2} \eta_{2},
$$

where $\xi_{1}, \xi_{2}, \eta_{1}$ and $\eta_{2}$ are fixed integers while $m_{1}$ and $m_{2}$ are arbitrary parameters. Substituting the values of $p, q, x_{1}, x_{2}, y_{1}$ and $y_{2}$ in (9.5)-(9.7), we get three simple equations

$$
m_{1}^{3} C_{i}^{\prime}\left(\xi_{1}, \xi_{2}\right)=m_{2}^{3} C_{i}^{\prime}\left(\eta_{1}, \eta_{2}\right), \quad i=1,2,3,
$$

and if these equations are simultaneously satisfied for certain integer values of $m_{1}$ and $m_{2}$, we obtain a solution of (VIII). Thus, a necessary and sufficient 
condition for the solvability of (VIII) is that there exist rational numbers $p$ and $q$ such that the discriminant $\phi(p, q)$ obtained above is a perfect square and, at the same time, the resulting equations (9.18) have a solution in integers for $m_{1}$ and $m_{2}$.

As an example, we consider the diophantine system

$$
\begin{aligned}
& x_{1}^{3}+x_{2}^{3}=y_{1}^{3}+y_{2}^{3}, \\
& x_{1}^{3}+x_{3}^{3}=y_{1}^{3}+y_{3}^{3}, \\
& x_{1} x_{2} x_{3}=y_{1} y_{2} y_{3} .
\end{aligned}
$$

Since this system is unaltered if we interchange $x_{2}, x_{3}$ as well as $y_{2}, y_{3}$, we need to search only for solutions with $x_{1} \neq y_{1}$ and $x_{1} y_{2}-x_{2} y_{1} \neq 0$. We write $x_{3}=p x_{1}+q x_{2}, y_{3}=p y_{1}+q y_{2}$, and obtain three equations of the type $\left(\mathrm{VIII}^{\prime}\right)$. If we take either $p=0$, or $q=0$, we obtain solutions that can only be considered as trivial solutions of (9.19), for instance, of the type $\left(x_{1}, x_{2}, x_{3}, y_{1}, y_{2}, y_{3}\right)=(0, \alpha, \alpha, \alpha, 0,0)$ where $\alpha$ is arbitrary. When $p q \neq 0$, the three equations of type $\left(\mathrm{VIII}^{\prime}\right)$ are linearly independent, and proceeding as above, we obtain an equation of the type (9.16) which in this case reduces to

$$
p^{2} x_{1}^{2}+p q x_{1} x_{2}+q^{2} x_{2}^{2}=0 .
$$

The only rational solution of this equation with $p q \neq 0$ is $x_{1}=0, x_{2}=0$, which leads to trivial solutions of (9.19). It follows that (9.19) has no essentially non-trivial solutions.

\section{References}

[1] A. Choudhry, Symmetric diophantine systems, Acta Arith. 59 (1991), 291-307.

[2] - Triads of cubes with equal sums and equal products, Math. Student 70 (2001), $137-143$.

[3] -, On the solvability of two simultaneous symmetric cubic diophantine equations with applications to sextic diophantine equations, Rocky Mountain J. Math. 32 (2002), 91-104.

[4] R. K. Guy, Unsolved Problems in Number Theory, 2nd ed., Springer, New York, 1994.

[5] L. J. Mordell, Diophantine Equations, Academic Press, London, 1969.

D-6/1, Multi-Storey Flats, Sector 13, R. K. Puram

New Delhi-110066, India

E-mail: ajaic203@yahoo.com

Received on 13.12.2004

and in revised form on 17.6.2005 